\title{
Glioblastoma, IDH-Wildtype
}

National Cancer Institute

\section{Source}

National Cancer Institute. Glioblastoma, IDH-Wildtype. NCI Thesaurus. Code C39750.

A glioblastoma that arises de novo. It is more commonly seen in older patients.

Mutations in IDH1 or IDH2 genes are not present. 